\title{
Panorama mundial das tecnologias referentes ao uso do ejiao por meio de busca em
}

\section{base de patentes}

\author{
Worldwide panorama of technologies related to the use of ejiao through patent search \\ Panorama mundial de tecnologías relacionadas con el uso de ejiao mediante búsqueda basada en
} patentes

Recebido: 27/01/2021 | Revisado: 02/02/2021 | Aceito: 09/02/2021 | Publicado: 14/02/2021

Lucas Santana da Fonseca

ORCID: https://orcid.org/0000-0002-5261-9695 Universidade Federal de Alagoas, Brasil E-mail: lucasfonseca1989@gmail.com

Cláudia Beatriz Lopez Almeida

ORCID: https://orcid.org/0000-0003-4750-6896 Universidade Federal de Alagoas, Brasil E-mail: claudiabeatrizla@gmail.com

Tobyas Maia Albuquerque Mariz ORCID: https://orcid.org/0000-0002-9078-8756 Universidade Federal de Alagoas, Brasil E-mail: tobyasmariz@hotmail.com

Chiara Albano Araújo Oliveira ORCID: https://orcid.org/0000-0003-4970-1070 Universidade Federal da Bahia, Brasil E-mail: oliveirachiara@gmail.com

Pierre Barnabe Escodro

ORCID: https://orcid.org/0000-0002-9409-660X Universidade Federal de Alagoas, Brasil E-mail: pierre.vet@gmail.com

\section{Resumo}

O colla corii asini ou donkey-hide gelatin ou ejiao é uma gelatina obtida do colágeno da pele de jumentos, utilizado há milhares de anos com diversas finalidades na medicina tradicional chinesa. A demanda pelo ejiao, misturado a ervas e outros ingredientes da medicina chinesa, aumentou devido a ascensão econômica da população chinesa em geral. As peles de jumentos oriundas de países como o Quirguistão, o Brasil e o México seguem para a China para a produção do Ejiao. Nessa cadeia extrativista a África é o território mais afetado, tanto no número de animais abatidos quanto no impacto social e econômico. Este trabalho é um levantamento prospectivo de patentes sobre inovações e descobertas do uso terapêutico do ejiao. A pesquisa foi realizada em três bases de patentes e a partir destas foram recuperados o total de 1837 documentos sobre tecnologias que fazem uso do ejiao. Analisando a trajetória temporal das publicações na área, observa-se que as invenções tiveram início na década de 90 e atingiram seu ápice no ano de 2015, contudo observou-se uma recaída nos anos seguintes, a suposição é de que o declínio tenha sido ocasionado pela redução populacional dos asininos na China e nos países fornecedores de outras regiões do mundo, além não existência da cadeia produtiva de pele de jumentos.

Palavras-chave: Jumentos; Colla corii asini; Donkey-hide gelatin.

\begin{abstract}
Colla corii asini or donkey-hide gelatin or ejiao is a gelatin obtained from the collagen of donkeys' skin, used for thousands of years for various purposes in traditional Chinese medicine. Demand for ejiao, mixed with herbs and other ingredients in Chinese medicine, has increased due to the economic rise of the Chinese population in general. The donkey skins from countries like Kyrgyzstan, Brazil and Mexico go to China to produce Ejiao. In this extractive chain, Africa is the most affected territory, both in the number of animals slaughtered and in the social and economic impact. This work is a prospective patent survey on innovations and discoveries in the therapeutic use of ejiao. The research was carried out on three patent bases and from these the total of 1837 documents on technologies that make use of ejiao were recovered. Analyzing the temporal trajectory of publications in the area, it is observed that inventions started in the 90s and reached their peak in 2015, however there was a relapse in the following years, the assumption is that the decline was caused by the population reduction of donkeys in China and in the supplier countries of other regions of the world, in addition to the non-existence of the donkey skin production chain.
\end{abstract}

Keywords: Donkey; Colla corii asini; Donkey-hide gelatin. 


\begin{abstract}
Resumen
Colla corii asini o gelatina de piel de burro o ejiao es una gelatina obtenida del colágeno de piel de burro, utilizada durante miles de años para diversos fines en la medicina tradicional china. La demanda de ejiao, mezclado con hierbas y otros ingredientes de la medicina china, ha aumentado debido al aumento económico de la población china en general. Las pieles de burro de países como Kirguistán, Brasil y México van a China para producir Ejiao. En esta cadena extractiva, África es el territorio más afectado, tanto en el número de animales sacrificados como en el impacto social y económico. Este trabajo es una encuesta prospectiva de patentes sobre innovaciones y descubrimientos en el uso terapéutico del ejiao. La investigación se realizó sobre tres bases de patentes y de estas se recuperaron un total de 1837 documentos sobre tecnologías que hacen uso del ejiao. Analizando la trayectoria temporal de las publicaciones en la zona, se observa que los inventos comenzaron en la década de los 90 y alcanzaron su pico en el 2015, sin embargo hubo una recaída en los años siguientes, el supuesto es que el descenso fue provocado por la reducción poblacional de burros. en China y en los países proveedores de otras regiones del mundo, además de la inexistencia de la cadena de producción de piel de burro.
\end{abstract}

Palabras clave: Burros; Colla corii asini; Gelatina de piel de burro.

\title{
1. Introdução
}

A colla corii asini ou donkey-hide gelatin, é uma gelatina extraída da pele da espécie Equus asinus, também chamada de ejiao. O produto é utilizado há mais de 2.000 anos na medicina tradicional chinesa nas terapias anti anêmia e antihemorrágica, além de consumido como alimento nutritivo que promove a saúde e vitalidade (Lv et al., 2011; Wu et al., 2007).

A partir do ano de 1980 vários estudos foram realizados buscando a composição química do ejiao, destacando-se principalmente proteínas (colágenos e albumina), mais de 20 tipos diferentes de aminoácidos (Liu et al., 1983; Chen et al. 1991; Wang et al., 2014), diversas substâncias inorgânicas, substâncias voláteis e polissacarídeos (Wang et al., 2014). Nesse período foram realizados estudos das ações farmacêuticas do produto, existindo estudos na tentativa de comprovação de sua eficácia frente a tumores (Liu et al., 2005; Li et al., 2013), processos inflamatórios (Chen, BF, 2011; Liu et al., 2019) e doenças hematológicas (Wu et al. 2007; Deng et al., 2011; Liu et al., 2014). Também são reportados resultados da ação do ejiao como tônico revigorante anti-fadiga (Song et al., 2011; Nongxuan, 2016), atividade imunomoduladora (Chengbiao e Qiusheng, 1991; Ying et al. 2011) e até como antioxidante com potencial anti-idade (Wang et al., 2012).

Com a tradição da medicina chinesa apoiando-se em descobertas de componentes do ejiao e suas possíveis propriedades, houve considerável aumento de interesse da população, principalmente asiática, pelo produto o que gerou crescimento exponencial do seu consumo. Visto isso, a população de jumentos da China, e dos países com grandes populações de jumentos, encontram-se ameaçadas pela exploração desenfreada da pele desses animais. A demanda atual deste mercado está em torno de 4 a 10 milhões de jumentos por ano, tendo, a espécie, cerca de 44 milhões de indivíduos por todo o mundo (Köhle, 2018). Na China houve uma redução drástica do plantel, que era de 11 milhões de jumentos nos anos 90 e passou a apenas 6 milhões em 2016. No Brasil a população de jumentos está estimada em 376.874 exemplares, com 326.569 na região Nordeste (IBGE, 2017). O abate indiscriminado da espécie tem chamado a atenção no país.

Este trabalho teve como objetivo o levantamento prospectivo de patentes sobre inovações e descobertas do uso terapêutico do Ejiao, diante deste cenário de aumento de sua produção e consumo nos últimos anos.

\section{Metodologia}

Este estudo foi focado na avaliação retrospectiva descritiva, de caráter exploratório, realizando-se primeiramente uma pesquisa preliminar buscando a verificação da aderência dos termos, bem como o comportamento das bases de dados de patentes aos termos e às strings formadas. Conforme Pereira et al. (2018) o método quantitativo utiliza das métricas para projetar previsões dos fatos estudados. 
Foram realizadas buscas na base do Instituto nacional de propriedade industrial - INPI e nas internacionais "Derwent" e do escritório europeu, a Espacenet. Nestas foram testados os termos que se apresentaram como aqueles que representam a temática (Quadro 1):

Quadro 1 - Termos utilizados na busca preliminar sobre inovações e descobertas do uso terapêutico do ejiao em bases de patentes nacionais e internacionais.

\begin{tabular}{|l|l|l|}
\hline Termos & Base de patente & Resultados \\
\hline Ejiao & INPI & 0 \\
\hline Colla corii asini & INPI & 0 \\
\hline Donkey-hide gelatin & INPI & 0 \\
\hline Ejiao & ESPACENET & 15 \\
\hline Colla corii asini & ESPACENET & 1640 \\
\hline Donkey-hide gelatin & ESPACENET & 3759 \\
\hline Ejiao & Derwent & 63 \\
\hline Colla corii asini & Derwent & 2960 \\
\hline Donkey-hide gelatin & Derwent & 3443 \\
\hline
\end{tabular}

INPI: é uma autarquia federal vinculada ao Ministério da Economia. Cuja missão é estimular a inovação e a competitividade a serviço do desenvolvimento tecnológico e econômico do Brasil, por meio da proteção eficiente da propriedade industrial;

Espacenet: é um serviço online gratuito para pesquisar patentes e pedidos de patentes;

Derwent: é um banco de dados que contém pedidos de patentes e subsídios.

Fonte: Autores (2019).

A base nacional INPI não apresentou resultados sobre o ejiao (Quadro 1), desta maneira foi excluído seu uso da pesquisa. No entanto, diversos questionamentos podem ser levantados sobre o motivo de não haverem invenções nesta área depositadas no Brasil. Após a realização de uma segunda busca nas bases de patentes, onde foi adotado o corte temporal de patentes depositadas de 1990 até a data da pesquisa (junho/2019), observando-se uma pequena variação nos resultados e constatando-se que este intervalo concentrou a maior parte dos depósitos nesta área (Quadro 2). 
Quadro 2 - Organização da busca com o corte temporal em bases de patentes sobre inovações e descobertas do uso terapêutico do ejiao.

\begin{tabular}{|l|l|l|l|}
\hline Termo & data & base & Resultado \\
\hline Ejiao & $01 / 01 / 1990$ até junho/2019 & ESPACENET & 15 \\
\hline Colla corii asini & $01 / 01 / 1990$ até junho/2019 & ESPACENET & 1640 \\
\hline Donkey-hide gelatina & $01 / 01 / 1990$ até junho/2019 & ESPACENET & 3756 \\
\hline Ejiao & $01 / 01 / 1990$ até junho/2019 & Derwent & 63 \\
\hline Colla corii asini & $01 / 01 / 1990$ até junho/2019 & Derwent & 2960 \\
\hline Donkey-hide gelatin & $01 / 01 / 1990$ até junho/2019 & derwent & 3441 \\
\hline
\end{tabular}

INPI: é uma autarquia federal vinculada ao Ministério da Economia. Cuja missão é estimular a inovação e a competitividade a serviço do desenvolvimento tecnológico e econômico do Brasil, por meio da proteção eficiente da propriedade industrial;

Espacenet: é um serviço online gratuito para pesquisar patentes e pedidos de patentes;

Derwent: é um banco de dados que contém pedidos de patentes e subsídios.

Fonte: Autores (2019).

Finalizando a fase de busca, foi confeccionado a string "ejiao or 'colla corii asini' or 'donkey-hide gelatin"”, já que os termos apresentaram aderência na pesquisa, juntamente com corte temporal. Vale salientar que em cada base de patentes houve pequenas adequações da string, já que em cada um destes bancos de dados os campos de busca se comportam de maneira diferente.

A segunda fase da pesquisa consistiu na seleção dos resultados obtidos, utilizando-se os seguintes critérios de exclusão: i) patentes duplicadas, provindas do cruzamento dos resultados de duas bases; ii) patentes que estavam fora do contexto da pesquisa; e por fim, iii) patentes que tivessem as informações disponíveis (Quadro 3). Sobre este último vale salientar que algumas bases possuem limitação na quantidade de resultados que podem ser resgatados, como o Espacenet, que disponibilizam apenas os 500 primeiros resultados.

Quadro 3 - Busca com o corte temporal em bases de patentes sobre inovações e descobertas do uso terapêutico do ejiao.

\begin{tabular}{|l|l|l|l|}
\hline String de busca & Data & Base & Resultado \\
\hline Ejiao or "Colla corii asini" or "Donkey-hide gelatin" & $01 / 01 / 1990$ até junho/2019 & ESPACENET & 5399 \\
\hline Ejiao or "Colla corii asini" or "Donkey-hide gelatin" & $01 / 01 / 1990$ até junho/2019 & derwent & 6301 \\
\hline
\end{tabular}

INPI: é uma autarquia federal vinculada ao Ministério da Economia. Cuja missão é estimular a inovação e a competitividade a serviço do desenvolvimento tecnológico e econômico do Brasil, por meio da proteção eficiente da propriedade industrial;

Espacenet: é um serviço online gratuito para pesquisar patentes e pedidos de patentes;

Derwent: é um banco de dados que contém pedidos de patentes e subsídios.

Fonte: Autores (2019).

Para finalizar, foi realizada a etapa da análise de dados, onde procurou-se examinar os dados das patentes referentes aos principais países depositantes, a trajetória temporal dos depósitos, os principais autores na área e as principais aplicações 
do Ejiao, utilizando a classificação internacional das patentes, inter-relacionando com as informações obtidas por órgão responsáveis como The Donkey Sanctuary e os artigos levantados como referência na temática.

\section{Resultados e Discussão}

Após as pesquisas e o agrupamento dos resultados de cada base, foram recuperados o total de 1837 documentos sobre tecnologias que fazem uso do ejiao. Analisando a trajetória temporal das publicações na área, observa-se que as invenções associadas ao ejiao tiveram início na década de 90 e atingiram seu ápice recentemente no ano de 2015. Contudo verifica-se uma queda nos anos seguintes (Figura 1). Sugere-se que este declínio tenha sido ocasionado pela redução da população de jumentos na China e outras regiões do mundo que são fornecedoras dos animais, além da não existência da cadeia produtiva de jumentos para o abate que existe apenas na China, conforme aborda o relatório Sob a pele (The Donkey Sanctuary, 2017).

Figura 1 - Trajetória temporal das patentes publicadas em duas bases sobre tecnologias que utilizam ejiao.

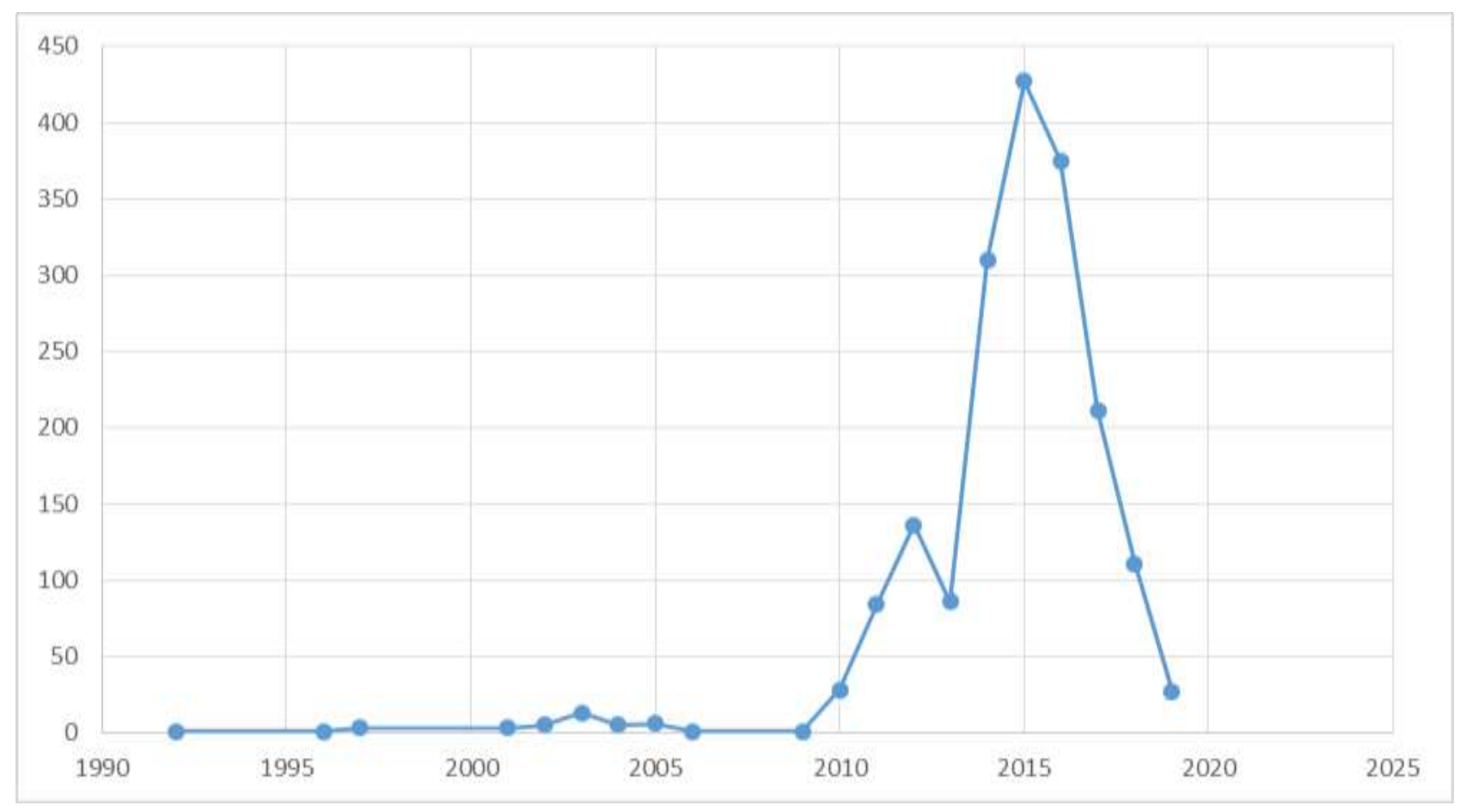

Bases utilizadas para as buscas de documentos sobre tecnologias que utilizam o Ejiao.

Fonte: Autores (2019).

A primeira patente publicada na área, foi chinesa e ocorreu no ano de 1992, descrevendo uma tecnologia focada no tratamento de câncer, composta por: ejiao, EXD-7-oxi-heterodiciclo $(2,2,1)$ heptano-2,3-carboxianidrido - OHD e raiz de alcaçuz. Já a última publicação foi em 2019 relativa a uma pasta oral, que possui o ejiao como componente, utilizada para o tratamento de problemas ocasionados pela gripe. Nota-se que as patentes mencionadas, utilizam das atividades terapêuticas do colla corii asini mas sempre associado a outros componentes.

Em relação a jurisdição, os pedidos são majoritariamente da China, sendo ínfima a aparição de outros países depositantes (Figura 2). Antigamente para os chineses o ejiao era considerado um produto para os nobres, mas com a ascensão econômica da classe média chinesa o comércio desse tipo de produto vem crescendo, é a população em busca de seus inúmeros benefícios para a saúde (The Sanctuary Donkey, 2017). 
Research, Society and Development, v. 10, n. 2, e25210212428, 2021

(CC BY 4.0) | ISSN 2525-3409 | DOI: http://dx.doi.org/10.33448/rsd-v10i2.12428

Figura 2 - Jurisdição dos pedidos de patentes de produtos com ejiao.

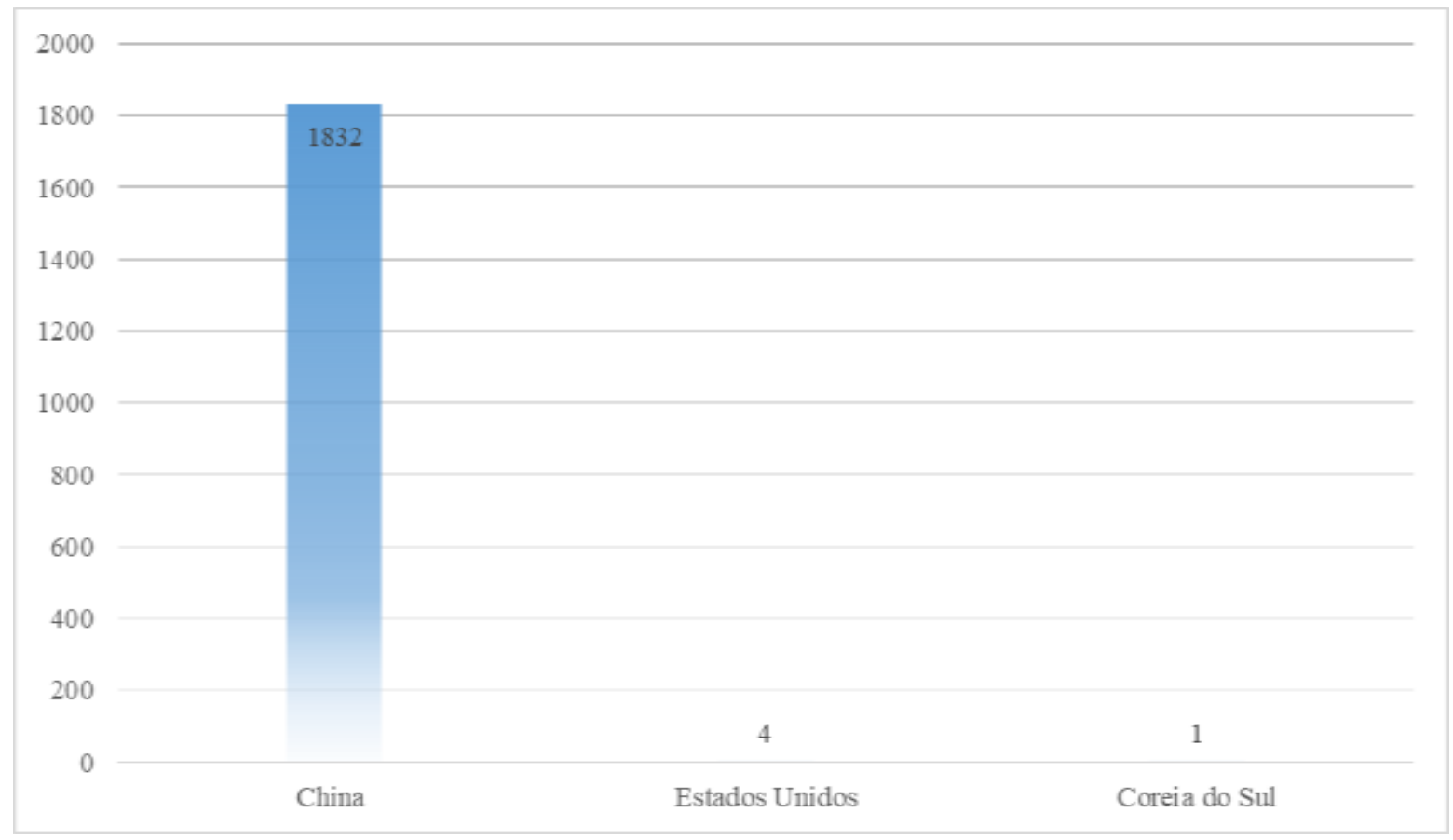

Bases de busca: INPI, Derwent e Espacenet.

Fonte: Autores (2019).

Nas aplicações das tecnologias que utilizam o Colla corii asini (Figura 3), observa-se a concentração dos pedidos na seção A (Necessidades humanas), conseguinte a subseção A61 (Ciência médica ou Veterinária; Higiene) que é a classificação com o maior número de patentes verificadas na pesquisa, no caso a A61K 35/00 destinada a preparações medicinais contendo materiais de constituição indeterminada.

Na sequência está a classificação A23L 33/00 focada em modificações das qualidades nutritivas de alimentos, produtos dietéticos, seu preparo ou tratamento; ainda a extinta classificação A23L 1/00 relacionada a alimentos sua preparação ou tratamento. De acordo com Wang et al (2014) a composição do ejiao, formada por aminoácidos, proteínas e polissacarídeos, entre outros, a relação entre estes constituintes químicos e moléculas bioativas incentivam a produção de pesquisas e desenvolvimento de produtos nutraceuticos e terapeuticos. 
Figura 3 - Principais classificações sobre tecnologias que fazem uso do ejiao

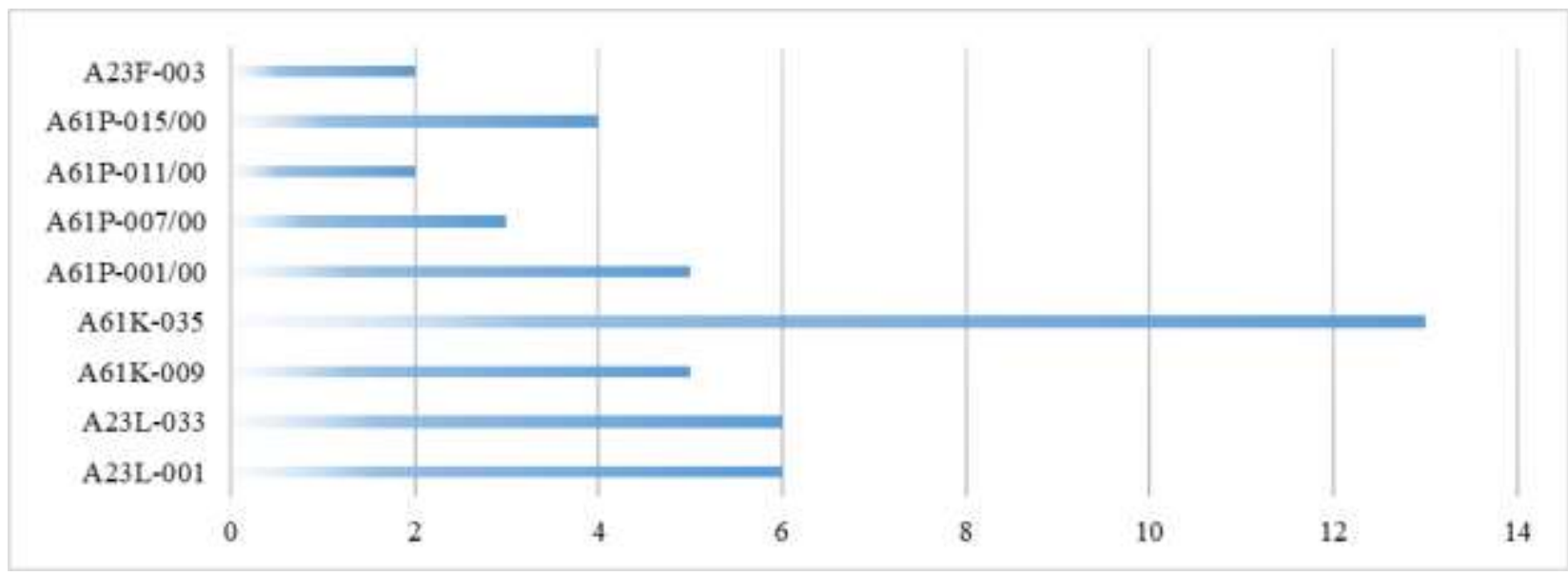

A61 (Ciência médica ou Veterinária; Higiene).

A23 (Necessidades Humanas - Qualidade nutritiva de alimentos).

A61 (Ciência médica ou Veterinária; Higiene - preparações para fins médicos, dentários ou de toalete.

Fonte: Autores (2019).

Sobre os principais depositantes na área (Figura 4), vê-se uma grande participação de inventores independentes chineses como Zhang, Wang, Chen, Wang, Chen, sendo Li C o principal depositante na área. Uma de suas patentes retrata uma composição para o tratamento de leucemia, que inclui entre os componentes o Colla corii asini.

Figura 4 - Principais depositantes de pesquisas realizadas com a utilização do ejiao.

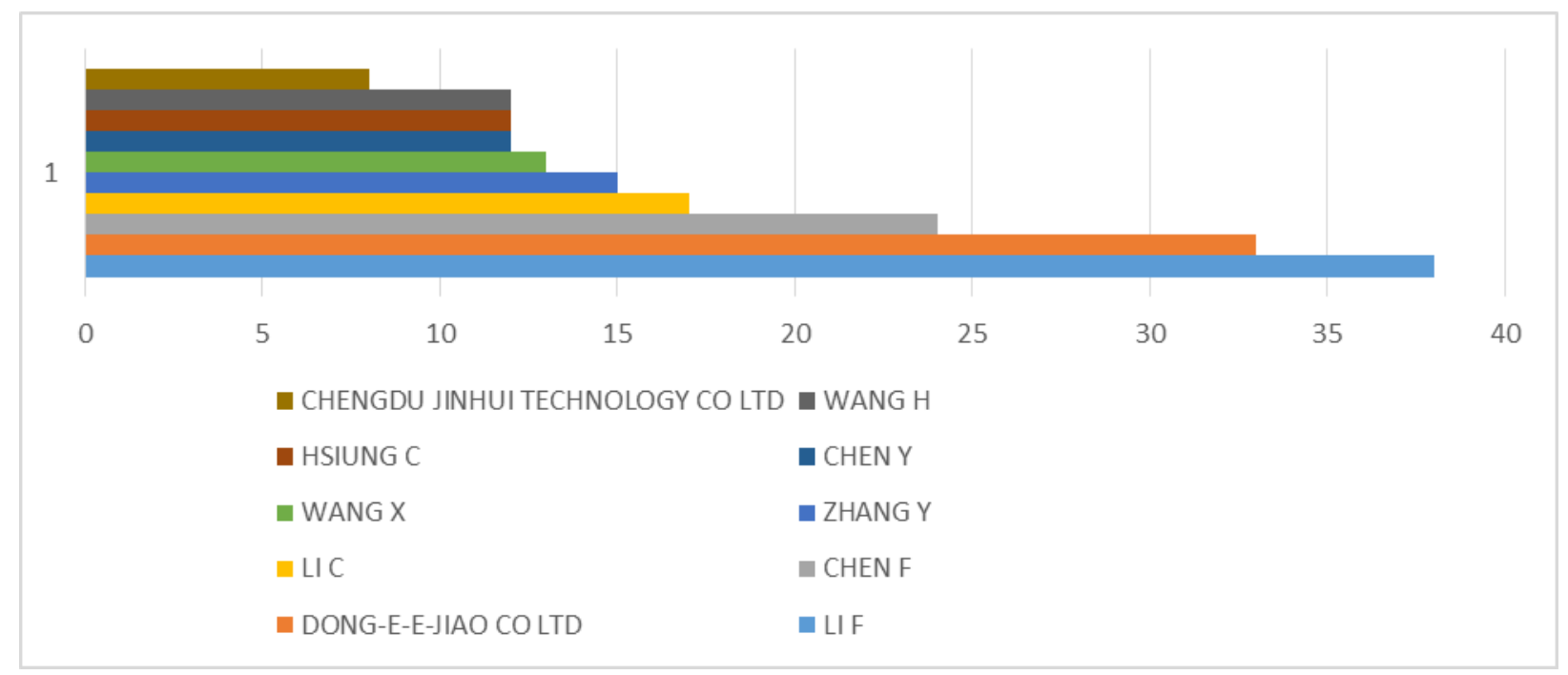

Indicar autores independentes e empresas

Fonte: Autores (2019).

Empresas chinesas especializadas no ejiao, também aparecem como principais depositantes, a exemplo da Dong-E-Ejiao Co LTD, antigamente chamada de Shan Dong Dong-E E-jiao Co, foi fundada em 1952, operando no mercado regional, focada na pesquisa, desenvolvimento, produção e venda de medicamentos tradicionais chineses usando Ejiao (Reuters, 2019). Como exemplo de suas patentes, pode ser citada a invenção de uma bebida com objetivo de nutrir e suplementar o colágeno da pele.

Analisando as informações, é visível a força deste produto no mercado chinês, o que vem movimentando o abate de jumentos globalmente (The Sanctuary Donkey, 2017). Segundo Carneiro et al. (2018), a população de jumentos na América do 
Sul teve um declínio entre 1996 e 2016 onde o Brasil apresentou uma redução populacional do animal de 37,08\%, para atender a demanda pela pele dos asnos, torando-se necessário a criação de políticas para construção de cadeias produtivas para evitar a extinção dos animais.

\section{Considerações Finais}

O país que mais produziu ciência envolvendo o ejiao foi a China seguido dos Estados Unidos da América. O Brasil não produziu patentes sobre o assunto. Quanto ao desenvolvimento científico ao longo dos últimos 10 anos, observou-se um crescimento gradual, com pico em 2015 com queda nos anos seguintes. Em relação às áreas de maior interesse, destacaram-se necessidades humanas, seguida da ciência médica ou veterinária e higiene. Os achados deste estudo reforçam a necessidade de compilar mais estudos para esclarecerimentos sobre o tema.

\section{Referências}

Carneiro G. F., Lucena, J. E. C., \& Barros, L. O. (2018). The current Situation and Trend of the Donkey Industry in South America. Journal of equine veterinary science; $65,106-110$.

Chen, B. F. (2011). Clinical observation on triple therapy of Ejiao and Weifuchun for chronic atrophic gastritis combined with peptic ulcer. International Journal of Clinical and Experimental Medicine; 10, 1622-1623.

Chen, D. Y., Wang, J. Z., \& Liu, W. L. (1991). Analytical studies on amino acids and trace elements in donkey-hide gelatin. China journal of Chinese materia medica; 16(2), 83-84.

Chengbiao, L., \& Qiusheng, T. (1991) The Effect of EJIAO on Cellular Immunological Function of Normal Mice. Pharmacology and Clinics of Chinese Materia Medica; 4.

Deng, W. L., Wu, H. Z., Xu, W., Zhang, Y. X., \& Lu, M. (2011). Effective component of colla corri asini on blood anemia induced by cyclophosphamide in mice bone marrow microenviroment. Lishizhen. Medicine and Materia Medica Research; 22, 2542-2544.

Instituto Brasileiro de Geografia e Estatística (IBGE). Censo agro 2017.

Köhle, N. (2018). Feasting on the dunkey skin. In: Prosperity. Golley J. \& Jaivin L. Acton ACT, Australia: ANU Press, pag. 176-181.

Li, N., Chen, XI., Li, X., Zhang, D., Chu, Z., \& Feng, J. (2013). Clinical observation in treating cancer-related fatigue with Fufang Ejiao Jiang. China Journal of Traditional Chinese Medicine and Pharmacy. 28(2).

Liu, C. L. (1983). Ingredients of colla corii asini comparison analysis research. Chin Tradit Pat Med; 1, 36-37.

Liu, M., Tan, H., Zhang X., Liu, Z., Cheng, Y., \& Wang, F. (2014). Hematopoietic effects and mechanisms of Fufang e'jiao jiang on radiotherapy and chemotherapy-induced myelosuppressed mice. J_Ethnopharmacol; 152(3), 575-84.

Liu, P. M., You, J. H., Xie, F. S., Cai, B. C., Xie, X. J., \& Tian, S. S. (2005). Experimental research of donkey-hide gelatin on restraining tumor and prolonging survival time of tumor carrying mice. Chin Med Sci Heal; 2, 25-26.

Liu, T., Zhang, P., Ling, Y., Hu, G., Gu, J., Yang, H., Wei, J., Wang, A., \& Jin, H. (2019). Protective Effect of Colla corii asini against Lung Injuries Induced by Intratracheal Instillation of Artificial Fine Particles in Rats. International Journal of Molecular Sciences; $20(1), 55$.

Lv, P., Zhao, Y. J., Qi, F., Zhou, X. S., You, J. H., Qin, Y. F., \& Zhang, Y. X. (2011). Authentication of equine DNA from highly processed donkey-hide glue (Colla corii asini) using sine element. J Food Drug Anal; 19, 123-130.

Nongxuan, M. A. O. (2016). The Anti-Fatigue Effect Of Compound Donkey-Hide Gelatine Mucilage On Free Combat Athletes. Journal Of Computational And Theoretical Nanoscience, 13(3), 20912095.

Pereira, A. S., et al. (2018). Metodologia da pesquisa científica. UFSM.

Song, Y. M., Mao, G. N., Huang, X. S., \& Dou, Y. M. (2011). Study on hemopoiesis and anti-fatigue effects of colla corri asini effervescent granules in mice. Prog Vet Med; 32, 83-86.

Wang, D. L., Ru, W. W., Xu, Y. P., Zhang, J. L., He, X. X., Fan, G. H., Mao, B., Zhou, X., \& Qin, Y. F. (2014). Chemical constituents and bioactivities of Colla corii asini. Drug Discoveries \& Therapeutics; 8, 201-207.

Wang, D., Liu, M., Cao, J., Chng, Y., Zhuo, C., Xu, H., Tian, S., Zhang, Y., Zhang, J., \& Wang, F. 2012). Effect of Colla corii asini (E’jiao) on D-Galactose Induced Aging Mice. Biol. Pharm. Bull; 35, 2128-2132. 
Research, Society and Development, v. 10, n. 2, e25210212428, 2021

(CC BY 4.0) | ISSN 2525-3409 | DOI: http://dx.doi.org/10.33448/rsd-v10i2.12428

Wu, H. Z., Yang, F., Cui, S. Y., Qin, Y. F., Liu, J. W., \& Zhang, Y. X. (2007). Hematopoietic effect of fractions from the enzyme digested colla corii asini on mice with 5-fluorouracil induced anemia. Am J Chin Med. 35, 853-866.

Ying, J., Xiao, B., Yang, W., Zeng, X., Guo, J., \& Xiao, Y. (2011). Leukocytopoiesis-promoting Action Comparison of Effective Components from Caulis Spatholobi and Colla Corii Asini in Leukopenia Rats. Traditional Chinese Drug Research and Clinical Pharmacology; vol. 2. 